Pacific Journal of Mathematics

ON ALTERNATE RINGS AND THEIR ATTACHED JORDAN RINGS 


\title{
ON ALTERNATIVE RINGS AND THEIR ATTACHED JORDAN RINGS
}

\author{
Michael Rich
}

Let $A$ be an alternative ring and $A^{q}$ its attached quadratic Jordan ring. We show that if $A$ is finitely generated by $n$ generators then $A^{q}$ is finitely generated by the monomials in $A$ of degree $\leqq n+1$. It follows that if $A$ is finitely generated then $A$ is nilpotent if and only if $A^{q}$ is solvable, and for arbitrary $A$ the Levitzki radical of $A$ coincides with the Levitzki radical of $A^{q}$. Finally, if $A$ has an involution $*$ and $H(A, *)$ denotes the *-symmetric elements of $A$ then several results known for associative rings connecting properties of $H(A, *)$ to those of $A$ apply.

The Levitzki radical $L(R)$ of a ring $R$ (associative, Jordan, alternative) is known to be the maximal locally nilpotent ideal of $R$ and has the properties that $L(R)$ contains all locally nilpotent ideals of $R$ and that $L(R / L(R))=0$. In $[9,11]$ it is shown that if $R$ is an associative or alternative algebra over a commutative ring $\Phi$ such that $1 / 2 \in \Phi$ then $L(R)=L\left(R^{+}\right)$where $R^{+}$denotes the attached linear Jordan algebra. In $\$ 1$ we extend this by considering an alternative ring $A$ of arbitrary characteristic and its attached quadratic Jordan ring $A^{q}$. Recall that $A^{q}$ is defined to be the additive group of $A$ together with the quadratic operators $x^{2}$ and $U_{x}: a \mapsto x a x$ for all $x$ in $A$. The bilinear operators attached to these are $x \cdot y=x y+y x$ and $U_{x, y}: a \mapsto(x a) y+(y a) x=x(a y)+y(a x)$. The key result we prove is that if $A$ is generated by $x_{1}, x_{2}, \cdots, x_{n}$ then $A^{n+2} \subseteq A U_{A}$ and that $A^{q}$ is finitely generated by all monomials in $A$ of degree $\leqq n+1$. This enables us to conclude that $L(A)=L\left(A^{q}\right)$ and that if $A$ is finitely generated then $A$ is nilpotent if and only if $A^{q}$ is solvable.

In $\S 2$, we assume that $A$ is a ring with involution $*$ and note that several known results for associative rings in which $A$ inherits properties of $H(A, *)$ apply to alternative rings. In particular, if $A$ is alternative and if the quadratic Jordan ring $H(A, *)$ is nilpotent of index $n$ then $A$ is nil of index $\leqq 2 n$. Finally, if $A$ is an algebra over a field with at least $n$ elements and if $H(A, *)$ is nil of bounded index $n$, then $A$ is nil of bounded index $\leqq 2 n$.

1. Throughout we shall make use of the Moufang laws

$$
(x a x) y=x[a(x y)]
$$




$$
\begin{aligned}
y(x a x) & =[(y x) a] x \\
(x y)(a x) & =x(y a) x
\end{aligned}
$$

It is known that if $B, C$ are ideals of $A$ then $B U_{C}$ is an ideal of $A$. For if $b \in B, c \in C, a \in A$ then

$$
(c b c) a=c(b(c a))+(c a)(b c)-c(a b) c
$$

by (1) and (3). But $c(b(c a))+(c a)(b c)=b U_{c, c a} \in B U_{C}$ and $c(a b) c=$ $(a b) U_{c} \in B U_{C}$. Thus $\left(B U_{C}\right) A \subseteq B U_{C}$. Similarly $A\left(B U_{C}\right) \subseteq B U_{C}$. In particular $A U_{A}$ is an ideal of $A$.

LEMMA. If $u$ is a monomial in $A$ of degree $\geqq 2$ in $x$ and $u \neq x^{2}$ then either $u \equiv 0 \bmod A U_{A}$ or $u \equiv x^{2} y \bmod A U_{A}$ for some $y$ in $A$.

Proof. First note that $x^{2} y+y x^{2}=x U_{x, y} \in A U_{A}$ so that terms of the form $y x^{2}$ are covered by the Lemma. Now in view of the fact that $A U_{A}$ is an ideal of $A$ and that $(a b) c \equiv-(c b) a \bmod A U_{A}$, it follows that $\left(x^{2} a\right) b \equiv-(b a) x^{2} \bmod A U_{A}$ and $\left(a x^{2}\right) b \equiv-\left(x^{2} a\right) b \equiv(b a) x^{2} \bmod A U_{A}$. Similarly for their left-right duals: $b\left(a x^{2}\right) \equiv-x^{2}(a b) \bmod A U_{A}$ and $b\left(x^{2} a\right) \equiv x^{2}(a b) \bmod A U_{A}$. Thus, if we let $T_{a}=R_{a}$ or $T_{a}=L_{a}$, an easy induction on $s$ shows that if $u=x^{2} T_{a_{1}} T_{a_{2}} \cdots T_{a_{s}}$ then $u \equiv x^{2} y \bmod A U_{A}$ for some $y \in A$. It follows that if a factor of $u$ satisfies the results of the Lemma then so does $u$ itself.

We may assume now that $u$ has a factor $u^{\prime}$ which takes one of the forms:

(i) $\quad u^{\prime}=x T_{a_{1}} T_{a_{2}} \cdots T_{a_{k}} T_{x}$

or

(ii) $\quad u^{\prime}=\left(x T_{a_{1}} T_{a_{2}} \cdots T_{a_{k_{1}}}\right)\left(x T_{b_{1}} T_{b_{2}} \cdots T_{b_{k_{2}}}\right)$ for some $a_{1}, b_{1} \in A$.

For case (i) we induct on $k$ and note that the result is trivial for $k=1$. Assume then that the result holds for any $w=x T_{d_{1}} T_{d_{2}} \cdots T_{d_{n}} T_{x}$ with $d_{i} \in A$ and $n<k$. Now if for some $i T_{a_{t}}=R_{a_{1}}$ and $T_{a_{t+1}}=R_{a_{t+1}}$ then

$$
\begin{aligned}
u^{\prime} & =x T_{a_{1}} T_{a_{2}} \cdots T_{a_{k}} T_{x}=\left(\left(\left(x T_{a_{1}} \cdots T_{a_{t-1}}\right) a_{t}\right) a_{t+1}\right) T_{a_{1+2}} \cdots T_{a_{k}} T_{x} \\
& \equiv-\left[\left(a_{t+1} a_{t}\right)\left(x T_{a_{1}} T_{a_{2}} \cdots T_{a_{t-1}}\right)\right] T_{a_{t+2}} \cdots T_{a_{k}} T_{x} \quad \bmod A U_{A}
\end{aligned}
$$

so that $u^{\prime}=x T_{a_{1}} \cdots T_{a_{1-1}} L_{b} T_{a_{1+2}} \cdots T_{a_{k}} T_{x} \bmod A U_{A}$ for $b=-a_{1+1} a_{1 \cdot}$ By the induction hypothesis on the number of $T$ 's we have our result. Similarly if $T_{a_{i}}=L_{a_{1}}$ and $T_{a_{i+1}}=L_{a_{i+1}}$ for some $i$. Thus $T_{a_{2 m+1}}=$ $R_{a_{2 m+1}}$ and $T_{a_{2 m}}=L_{a_{2 m}}$ or $T_{a_{2 m+1}}=L_{a_{2 m+1}}$ and $T_{a_{2 m}}=R_{a_{2 m}}$ for all $m$. Therefore, if $k=2$ we have the cases $((a x) b) x,(a(x b)) x, x((a x) b)$, and $x(a(x b))$. But 


$$
((a x) b) x \equiv-(x b)(a x) \equiv-x(b a) x \equiv 0 \bmod A U_{A} \quad \text { by }
$$

and

$$
(a(x b)) x \equiv-(x(x b)) a \equiv-\left(x^{2} b\right) a \equiv(a b) x^{2} \bmod A U_{A}
$$

and similarly for the last two cases. Thus the result holds for $k=2$. Suppose now that $k>2$ and that $T_{a_{2 m+1}}=R_{a_{2 m+1}}$ and $T_{a_{2 m}}=$ $R_{a_{2 m}}$. Then

$$
u^{\prime}=\left[\left(a_{2}\left(x a_{1}\right)\right) a_{3}\right] T_{a 4} \cdots T_{a k} T_{x}
$$

Since $A$ is alternative we have $a_{2}\left(x a_{1}\right)=\left(a_{2} x\right) a_{1}+\left(a_{2} a_{1}\right) x-a_{2}\left(a_{1} x\right)$ so that

$$
u^{\prime}=x L_{a_{2}} R_{a_{1}} R_{a_{3}} T_{a 4} \cdots T_{a_{k}} T_{x}+x L_{a_{2} a_{1}} R_{a_{3}} T_{a_{4}} \cdots T_{a_{k}} T_{x}+x L_{a_{1}} L_{a_{2}} R_{a_{3}} T_{a 4} \cdots T_{a_{k}} T_{x}
$$

Since the the first term has two consecutive right multiplications, the last term has two consecutive left multiplications, and the middle term fewer than $k T$ 's, we have $u^{\prime}=x^{2}$, or $u^{\prime} \equiv 0 \bmod A U_{A}$, or $u^{\prime} \equiv x^{2} y \bmod A U_{A}$ for some $y$ by the induction hypothesis. If $T_{a_{2 m+1}}=L_{a_{2 m+1}}$ and $T_{a_{2 m}}=$ $L_{a_{2 m}}$ we get the same result using the fact that $\left(a_{1} x\right) a_{2}=$ $a_{1}\left(x a_{2}\right)-\left(x a_{1}\right) a_{2}+x\left(a_{1} a_{2}\right)$. Thus we have disposed of case (i).

For case (ii) we induct on $k=\min \left(k_{1}, k_{2}\right)$ and note that $k=0$ is case (i). If $k_{2} \leqq k_{1}$, we let $w=x T_{a_{1}} \cdots T_{a_{k_{1}}}, v=x T_{b_{1}} \cdots T_{b_{k_{2}-1}}$ and $c=b_{k_{2}}$ and we have one of the two cases:

$$
u^{\prime}=w(v c) \equiv-c(v w) \bmod A U_{A}
$$

or

$$
u^{\prime}=w(c v) \equiv-v(c w) \bmod A U_{A}
$$

Now if $k_{2}=k=1$ then $v w$ and $v(c w)$ are of the form of case (i) so that $u^{\prime}$ satisfies the results of the Lemma. If $k>1$ then both $v w$ and $v(c w)$ have a lower value of $k$, so by the induction hypothesis they satisfy the desired conclusion. Hence so does $u^{\prime}$. The case $k_{1} \leqq k_{2}$ follows from the left-right dual of (*). Thus, in all cases we get $u \equiv 0 \bmod A U_{A}$ or $u \equiv x^{2} y \bmod A U_{A}$ for some $y \in A$.

Theorem 1. If $A$ is generated by $n$ elements then $A^{n+2} \subseteq A U_{A}$.

Proof. Let $u \in A^{n+2}$. Then since $A$ has $n$ generators it follows that either there is at least one generator, say $x$, such that the degree of $u$ in $x$ is $\geqq 3$ or there are at least two generators, say $w$ and $z$, such that the degree of $u$ in $w$ is $\geqq 2$ and the degree of $u$ in $z$ is $\geqq 2$. If the latter 
holds then by the lemma if $u \neq 0 \bmod A U_{A}$ we have $u \equiv z^{2} y$ $\bmod A U_{A}$. Since $y$ is of degree at least two in $w$ we get $y=w^{2}$ or $y \equiv w^{2} a \bmod A U_{A}$ for some $a \in A$. Thus, either $u \equiv z^{2} w^{2} \bmod A U_{A}$ or $u \equiv z^{2}\left(w^{2} a\right) \bmod A U_{A}$. But $z^{2} w^{2} \equiv-w z^{2} w \equiv 0 \quad \bmod A U_{A} \quad$ and $z^{2}\left(w^{2} a\right) \equiv-a\left(w^{2} z^{2}\right) \equiv 0 \bmod A U_{A}$. Thus in this case $u \equiv 0 \bmod A U_{A}$.

If the former holds then $u \equiv x^{2} y \bmod A U_{A}$ where $y$ contains a factor $x$. Thus $u \equiv x^{2}\left(x T_{a_{1}} T_{a_{2}} \cdots T_{a_{k}}\right) \bmod A U_{A}$ for some $a_{\imath} \in A$. Thus $u \equiv 0 \bmod A U_{A}$ by induction on $k$. For if $k=1$ then we get $u \equiv x^{3} a_{1} \equiv$ $0 \bmod A U_{A}$ or $u \equiv x^{2}(a x) \equiv 0 \bmod A U_{A}$. As in the lemma we may assume that no two consecutive $T$ 's represent $R$ or $L$ so that the case $k=2$ reduces to $x^{2}\left(a_{2}\left(x a_{1}\right)\right)$ or $x^{2}\left(\left(a_{1} x\right) a_{2}\right)$. But $x^{2}\left(a_{2}\left(x a_{1}\right)\right)=$ $x\left[x\left(a_{2}\left(x a_{1}\right)\right)\right]=x\left[\left(x a_{2} x\right) a_{1}\right] \equiv 0 \quad \bmod A U_{A} \quad$ and $\quad x^{2}\left(\left(a_{1} x\right) a_{2}\right) \equiv$ $-a_{2}\left(\left(a_{1} x\right) x^{2}\right) \equiv 0 \bmod A U_{A}$. The inductive step is obtained precisely as in case (i) of the lemma. Thus $u \in A U_{A}$ and the theorem is proven.

REMARK. The advance in Theorem 1 is not the fact that a power of $A$ is contained in $A U_{A}$ but rather in the precise value $n+2$. For, as noted by Professor McCrimmon in a private communication, if $A$ is finitely generated then $\bar{A}=A / A U_{A}$ is finitely generated and nil satisfying the polynomial identity $x^{3}=0$. This, by an earlier result of his [6, Theorem 3] implies that $A$ is nilpotent so there is an integer $k$ such that $A^{k} \subseteq A U_{A}$.

THEOREM 2. If $A$ is generated by $x_{1}, x_{2}, \cdots, x_{n}$ then the Jordan ring $A^{q}$ is finitely generated by all monomials of degree $<n+2$.

Proof. Let $F$ be the free alternative ring generated by $x_{1}, x_{2}, \cdots, x_{n}$. Then if $u$ is an element of minimal degree in $A^{q}$ not generated by the monomials of degree $\leqq n+1$ then deg $u \geqq n+2$ so that

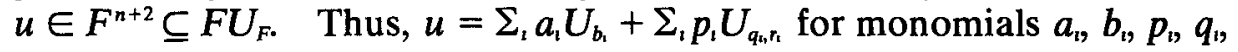
$r_{t}$ in $F$. Therefore $a_{t}, b_{t}, p_{t}, q_{1}, r_{t}$ have lower degree than $u$ and are generated in $F^{q}$ by the monomials of degree $<n+2$. Thus $u$ is generated by these monomials also and we have the result for $F$. Now $A^{q} \cong F^{q} / K$ for some ideal $K$ of $A^{q}$. Therefore $A^{q}$ is also generated by the monomials of degree $<n+2$.

Recall that if $J$ is a Jordan algebra then $D(J)=J U_{J}$ is a quadratic ideal of $J$, and the derived series of $J$ is given by

$$
J=D^{0}(J) \supset D(J) \supset D^{2}(J) \supset \cdots \supset D^{n}(J) \supset \cdots
$$

where $D^{i+1}(J)=D\left(D^{\prime}(J)\right) . \quad J$ is solvable if $D^{n}(J)=0$ for some $n$. The degree of an element is defined by $\operatorname{deg}\left(a U_{b}\right)=2 \operatorname{deg} b+\operatorname{deg} a$, $\operatorname{deg}\left(a U_{b, c}\right)=\operatorname{deg} a+\operatorname{deg} b+\operatorname{deg} c, \quad \operatorname{deg} a^{2}=2 \operatorname{deg} a$, and $\operatorname{deg} a \cdot b=$ $\operatorname{deg} a+\operatorname{deg} b . \quad J$ is nilpotent if there is an $n$ such that all monomials of 
degree $\geqq n$ are zero. McCrimmon has shown that if $J$ is finitely generated then $J$ is solvable iff $J$ is nilpotent [4]. In our situation we write $D^{t}(A)$ to denote $D^{t}\left(A^{q}\right)$.

COROLlary. If $A$ is finitely generated then for each $t$ there is a $k$ such that $A^{k} \subseteq D^{\prime}(A)$. Also $D^{\prime}(A)$ is finitely generated for every $t$.

Proof. The second statement follows immediately from Theorem 2, since it is known that if a Jordan algebra $J$ is finitely generated then so is $D^{t}(J)$ for all $t$ [4]. Thus, by Theorem $2, D^{t}(A)$ is finitely generated as a Jordan ring and hence, as an alternative ring. The first statement is arrived at by induction on $t$. The case $t=1$ is the statement of Theorem 1. Assume true for $t$. Since $D^{t}(A)$ is a finitely generated alternative ring then by Theorem 1 there is an integer $m$ such that $\left(D^{t}(A)\right)^{m} \subseteq$ $D\left(D^{t}(A)\right)=D^{t+1}(A)$. Thus $\left(A^{k}\right)^{m} \subseteq\left(D^{t}(A)\right)^{m} \subseteq D^{t+1}(A)$. By a result of Zwier [12] there is an integer $r$ such that $A^{r} \subseteq\left(A^{k}\right)^{m}$. Thus $A^{\prime} \subseteq D^{t+1}(A)$.

The following theorem extends a result of Shirshov for alternative algebras over a field of characteristic $\neq 2$.

THEOREM 3. If $A$ is a finitely generated alternative ring then $A$ is nilpotent iff $A^{q}$ is solvable iff $A^{q}$ is nilpotent.

Proof. Clearly, $A$ nilpotent implies $A^{q}$ solvable. The equivalence of $A^{q}$ solvable and $A^{q}$ nilpotent is the result of McCrimmon mentioned earlier. Since to each $t$ there is a $k$ such that $A^{k} \subseteq D^{t}(A)$ we conclude that $A^{q}$ solvable implies $A$ nilpotent.

Theorem 4. If $A$ is an alternative ring then $L(A)=L\left(A^{q}\right)$.

Proof. Clearly $L(A)$ is an ideal of $A^{q}$ and since it is locally nilpotent in $A$, it is also locally nilpotent in $A^{q}$. Thus $L(A) \subseteq L\left(A^{q}\right)$.

For the converse it is sufficient to prove that $L(A)=0$ implies that $L\left(A^{q}\right)=0$. For under this assumption if $L(A) \neq 0$ then, since $L(A / L(A))=0$, we get $L\left(A^{q} / L(A)\right)=0$. Since the homomorphic image of a locally nilpotent ideal is locally nilpotent we get $L\left(A^{q}\right) / L(A) \subseteq L\left(A^{q} / L(A)\right)=0$. Thus $L\left(A^{q}\right) \subseteq L(A)$.

Recall that if $B$ is an ideal of $A^{q}$ then $\operatorname{Ker} B=\{b \in B \mid b A+$ $A b \subseteq B\}$ is an ideal of $A$. It is shown in [5] that $A U_{B} \subseteq \operatorname{Ker} B$ and that $L(A)=0$ implies that $A$ is strongly semiprime in the sense that $A U_{a}=0$ implies that $a=0$. Assume now that $L(A)=0$ and that $L\left(A^{q}\right) \neq 0$. If Ker $L\left(A^{q}\right)=0$ then $A U_{L\left(A^{q}\right)}=0$ contradicting the fact that $A$ is strongly semiprime. Thus $L\left(A^{q}\right)$ contains a nonzero alternative ideal $K=$ $\operatorname{Ker} L\left(A^{q}\right)$. We show that $K \subseteq L(A)$ to obtain a contradiction. For if 
$R$ is a finitely generated alternative subring of $K$ then by Theorem $2 R^{q}$ is a finitely generated quadratic Jordan algebra. Since $R^{q} \subseteq L\left(A^{q}\right)$ it follows that $R^{q}$ is nilpotent. Then, by Theorem $3, R$ is a nilpotent ring. Thus $K$ is a locally nilpotent ideal of $A$ and $K \subseteq L(A)$ for the desired contradiction. It follows that $L(A)=0$ implies that $L\left(A^{q}\right)=0$ and the proof is complete.

REMARK. Note that the proof of Theorem 4 can be used equally well to show that the locally finite dimensional radical of $A$ coincides with the locally finite dimensional radical of $A^{q}$.

2. In the following let $A$ be an alternative ring with involution * and let $H(A, *)$ denote the Jordan ring of *-symmetric elements of $A$. In [3] McCrimmon asked the question: If $B$ is an associative algebra with involution $*$ such that all *-symmetric elements are nilpotent, does it follow that $B$ is itself necessarily nil? Osborn [8] answered the question in the affirmative if $B$ is an algebra over an uncountable field $\Phi$. In an analogous result Montgomery has shown that if $B$ is an associative algebra with involution over an uncountable field and if the symmetric elements of $B$ are algebraic then $B$ is algebraic [7]. We note that both of these results apply to an alternative algebra $A$ with involution. For if $a \in A$ then by Artin's theorem $A_{0}=\Phi\left[a, a^{*}\right]$ is an associative algebra. Since the symmetric elements of $A_{0}$ are nil (algebraic) it follows that $A_{0}$ is nil (algebraic). Thus the elements of $A$ are nilpotent (algebraic).

The key result needed by Osborn is the result of Amitsur that if $A$ is an associative algebra over a field $\Phi$ such that the cardinality of $\Phi$ exceeds the dimension of $A$ over $\Phi$ then the Jacobson radical of $A$ is nil ideal. We note that the proof of Amitsur's theorem as presented in [2, pp. 19-20] carries over verbatim to the alternative case once the following two observations are made. (1): the proof in [2] that the elements in the radical are either nilpotent or transcendental uses associativity but can be easily adjusted. For if $a \in \operatorname{Rad} A$ is algebraic then $\Phi[a]$ is finite dimensional. From the power-associativity of $A$ we know that $\Phi[a]$ is nil or contains an idempotent $e[10, \mathrm{p} .32]$. The latter implies that $e \in \operatorname{Rad} A$ which is impossible. Thus $a$ is nilpotent. (2): the proof of Proposition 2 in [2] requires the fact that $(a b) b^{-1}=a$ for all $a, b \in A$. This is also true in alternative rings [9, p. 38].

Some other results which relate nilpotency in $H(R, *)$ with nilpotency in $R$ for an associative ring $R$ are given in [9] under the assumption that $2 x=a$ is solvable for all $a$ in $R$. We note that these results also apply to an alternative ring $A$ with involution and do not require any characteristic assumptions. For the key result needed is that if $\widehat{\alpha \beta}(0,0)=1$ and $\widehat{\alpha \beta}(n, k)$ denotes the sum of all monomials of degree $n$ 
in $\alpha$ and degree $k$ in $\beta$, then for any $x \in R$ we get

$$
x^{2 n}=\left[\sum_{k=0}^{n-1} \widehat{\alpha \beta}(2 n-2 k-1, k)\right] x+\left[\sum_{k=0}^{n-1} \widehat{\alpha \beta}(2 k, n-k-1)\right] \beta
$$

for $\alpha=x+x^{*}$ and $\beta=-x^{*} x$. Since all of the computations take place in the subring generated by $x$ and $x^{*}$, by Artin's theorem this identity holds for an alternative ring $A$. Thus we get:

THEOREM. If $A$ is an alternative ring with involution $*$ and if the quadratic Jordan ring $H(A, *)$ is nilpotent of index $n$, then $A$ is nil of index $\leqq 2 n$.

Proof. As in [8], if $x \in A$ let $\alpha=x+x^{*}, \beta=-x^{*} x$. Then if $K_{x}$ denotes the quadratic Jordan subring of $H(A, *)$ generated by $\alpha$ and $\beta$ then $K_{x}$ is nilpotent of index $\leqq n$. If $K_{x}^{t}$ denotes the set of all sums of monomials in $K_{x}$ of degree $\geqq t$ then the proof of $[9$, Lemma 6] shows (without any characteristic assumptions) that $\widehat{\alpha \beta}(m, t) \in K^{m+t}$ for all $m, t$ such that $m+t \geqq 1$. Thus, by (4) $x^{2 n}=0$.

Corollary. If $H(A, *)$ is solvable then $A$ is a nil ring.

Proof. The proof of the previous theorem shows that if $x \in A$ and $K_{x}$ is nilpotent of index $n$ then $x^{2 n}=0$. Now since $H(A, *)$ is solvable it follows that $K_{x}$ is solvable. Since $K_{x}$ is finitely generated it is nilpotent of index $t$ for some $t$. Therefore $x^{2 t}=0$.

With our previous remarks the following theorem of [9] carries over to the alternative case with no changes.

THEOREM. Let $A$ be an alternative algebra with involution $*$ over a field $\Phi$ with at least $n$ elements. Then if $H(A, *)$ is nil with bounded nilindex $n, A$ is nil with bounded nilindex $\leqq 2 n$.

REMARK. In [9, theorem 3] it is shown that if $A$ is an associative algebra over a field $F$ of characteristic $\neq 2$ with involution then $L(H(A, *))=H(A, *) \cap L(A)$. We note that the same result holds for the locally finite dimensional radical $\mathscr{L}$. For, as in [9], the proof reduces to showing that if $U$ is a nonzero ideal of $A$ and $U \cap H(A, *) \subseteq$ $\mathscr{L}(H(A, *))$ then $U \subseteq \mathscr{L}(A)$. Assume then that $B$ is a finitely generated subalgebra of $U$. Then by the result of Osborn mentioned in [9], $H(B, *)$ is finitely generated and thus finite dimensional of dimension $n$ for some $n$. But then $H(B, *)$ is algebraic and satisfies a polynomial identity. Then, by a result of Baxter and Martindale [1], $B$ is finite dimensional. Thus, $U$ is a locally finite ideal of $A$ so that $U \subseteq \mathscr{L}(A)$. 


\section{REFERENCES}

1. W. E. Baxter and W. S. Martindale III, Rings with involution and polynomial identities, Canad. J. Math., 20 (1968), 465-473.

2. N. Jacobson, Structure of Rings, (Colloq. Publ. Vol. 37) Amer. Math. Soc., Providence (1968).

3. K. McCrimmon, On Herstein's theorems relating Jordan and associative algebras, J. Algebra, 13 (1969), 382-392.

4. — Solvability and nilpotence for quadratic Jordan algebras, Scripta Math., 29 (1973), $467-483$.

5. — Absolute zero divisors and local nilpotence in alternative algebras, Proc. Amer. Math. Soc., 47 (1975), 293-299.

6. — Alternative algebras satisfying polynomial identities, J. Algebra, 24 (1973), 283-292.

7. S. Montgomery, Algebraic algebras with involution, Proc. Amer. Math. Soc., 31 (1972), 368-372.

8. J. M. Osborn, Jordan and associative rings with nilpotent and invertible elements, J. Algebra, 15 (1970), 301-308.

9. M. Rich, The Levitzki radical in associative and Jordan rings, J. Algebra, 40 (1976), 97-104. 10. R. D. Schafer, An introduction to nonassociative algebra, Pure and Appl. Math., Vol. 22, Academic Press, New York, 1966.

11. A. M. Slinko, Radicals of Jordan rings connected with alternative rings, Math. Notes of Acad.

Sci. U.S.S.R., 16 (1974), 664-667.

12. P. J. Zwier, Prime ideals in a large class of nonassociative rings, Trans. Amer. Math. Soc., 158 (1971), 257-271.

Received August 16, 1976.

TEMPLE UNIVERSITY

PhILADELPHIA, PA 19121 


\section{PACIFIC JOURNAL OF MATHEMATICS}

\section{EDITORS}

RICHARD ARENS (Managing Editor)

University of California

Los Angeles, CA 90024

R. A. BeAumont

University of Washington

Seattle, WA 98105

C. C. MOORE

University of California

Berkeley, CA 94720
J. DUGUNDJI

Department of Mathematics

University of Southern California

Los Angeles, CA 90007

R. FINN AND J. MILGRAM

Stanford University

Stanford, CA 94305

\section{ASSOCIATE EDITORS}
E. F. BECKENBACH
B. H. NEUMANN
F. WOLF
K. YoshidA

\section{SUPPORTING INSTITUTIONS}

UNIVERSITY OF BRITISH COLUMBIA

UNIVERSITY OF SOUTHERN CALIFORNIA

CALIFORNIA INSTITUTE OF TECHNOLOGY

STANFORD UNIVERSITY

UNIVERSITY OF CALIFORNIA

UNIVERSITY OF HAWAII

MONTANA STATE UNIVERSITY

UNIVERSITY OF TOKYO

UNIVERSITY OF NEVADA

UNIVERSITY OF UTAH

NEW MEXICO STATE UNIVERSITY

OREGON STATE UNIVERSITY

UNIVERSITY OF OREGON

OSAKA UNIVERSITY

WASHINGTON STATE UNIVERSITY

UNIVERSITY OF WASHINGTON

AMERICAN MATHEMATICAL SOCIETY

The Supporting Institutions listed above contribute to the cost of publication of this Journal, but they are not owners or publishers and have no responsibility for its contents or policies.

Mathematical papers intended for publication in the Pacific Journal of Mathematics should be in typed form or offset-reproduced (not dittoed), double spaced with large margins. Underline Greek letters in red, German in green, and script in blue. The first paragraph or two must be capable of being used separately as a synopsis of the entire paper. Items of the bibliography should not be cited there unless absolutely necessary, in which case they must be identified by author and Journal, rather than by item number. Manuscripts, in duplicate, may be sent to any one of the four editors. Please classify according to the scheme of Math. Reviews, Index to Vol. 39. All other communications should be addressed to the managing editor, or Elaine Barth, University of California, Los Angeles, California, 90024.

100 reprints are provided free for each article, only if page charges have been substantially paid. Additional copies may be obtained at cost in multiples of 50 .

The Pacific Journal of Mathematics is issued monthly as of January 1966. Regular subscription rate: $\$ 72.00$ a year (6 Vols., 12 issues). Special rate: $\$ 36.00$ a year to individual members of supporting institutions.

Subscriptions, orders for numbers issued in the last three calendar years, and changes of address should be sent to Pacific Journal of Mathematics, 103 Highland Boulevard, Berkeley, California, 94708.

PUBLISHED BY PACIFIC JOURNAL OF MATHEMATICS, A NON-PROFIT CORPORATION

Printed at Jerusalem Academic Press, POB 2390, Jerusalem, Israel. 


\section{Pacific Journal of Mathematics}

\section{Vol. 75, No. $2 \quad$ October, 1978}

Susan Jane Zimmerman Andima and W. J. Thron, Order-induced

topological properties ................................... 297

Gregory Wade Bell, Cohomology of degree 1 and 2 of the Suzuki groups . . 319

Richard Body and Roy Rene Douglas, Rational homotopy and unique

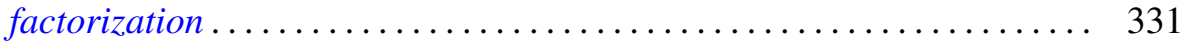

Frank Lewis Capobianco, Fixed sets of involutions ................. 339

L. Carlitz, Some theorems on generalized Dedekind-Rademacher sums .... 347

Mary Rodriguez Embry and Alan Leslie Lambert, The structure of a special class of weighted translation semigroups .....................

Steve Ferry, Strongly regular mappings with compact ANR fibers are

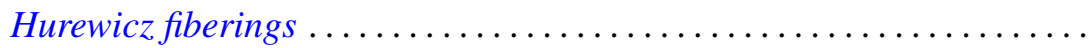

Ivan Filippenko and Marvin David Marcus, On the unitary invariance of the

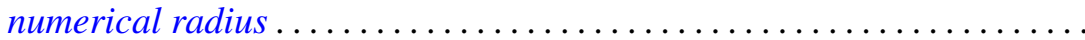

$\mathrm{H}$. Groemer, On the extension of additive functionals on classes of convex

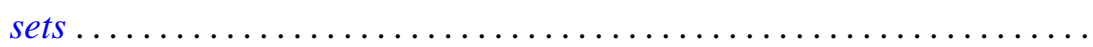

Rita Hall, On the cohomology of Kuga's fiber variety ............... 411

H. B. Hamilton, Congruences on $\mathrm{N}$-semigroups ................. 423

Manfred Herrmann and Rolf Schmidt, Regular sequences and lifting

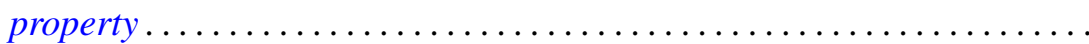

James Edgar Keesling, Decompositions of the Stone-Čech compactification which are shape equivalences .....................

Michael Jay Klass and Lawrence Edward Myers, On stopping rules and the expected supremum of $S_{n} / T_{n}$

Ronald Charles Linton, $\lambda$-large subgroups of $C_{\lambda}$-groups

William Owen Murray, IV and L. Bruce Treybig, Triangulations with the free cell property ............................

Louis Jackson Ratliff, Jr., Polynomial rings and $H_{i}$-local rings ...

Michael Rich, On alternate rings and their attached Jordan rings....

Gary Sampson and H. Tuy, Fourier transforms and their Lipschitz

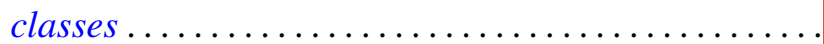

Helga Schirmer, Effluent and noneffluent fixed points on dendrites ...

Daniel Byron Shapiro, Intersections of the space of skew-symmetric maps

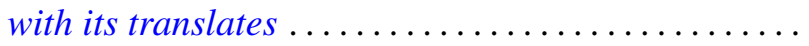

Edwin Spanier, Tautness for Alexander-Spanier cohomology ...

Alan Stein and Ivan Ernest Stux, A mean value theorem for binary digits ...

Franklin D. Tall, Normal subspaces of the density topology . .

William Yslas Vélez, Prime ideal decomposition in $F\left(\mu^{1 / p}\right) \ldots$ 